\title{
Ligand-side tautomer enumeration and scoring for structure-based drug-design
}

\author{
Thomas Seidel", G Wolber \\ From 5th German Conference on Cheminformatics: 23. ClC-Workshop \\ Goslar, Germany. 8-10 November 2009
}

Tautomeric rearrangements of a molecule lead to distinct equilibrated structural states of the same chemical compound that may differ significantly in molecular shape, surface, nature of functional groups, hydrogenbonding pattern and other derived molecular properties [1]. Especially for the structure-based pharmacophore modeling of ligand-protein complexes [2], knowledge of the most favorable tautomeric ligand states may be crucial for the quality and correctness of the generated pharmacophore models and derived putative binding modes. We will present a ligand-side tautomer enumeration and ranking algorithm that considers both geometrical constraints imposed by the conformation of the bound ligand as well as intra- and inter-molecular energetic contributions to find the preferred tautomeric states of the ligand in the macromolecular environment of the binding-site. The presented tautomer ranking algorithm is based on scores that are derived solely from MMFF94 [3-9] energies (thus the method works for a wide range of organic molecules) and has proven to be able to top-rank known preferred tautomeric states of ligands in a series of investigated protein complexes.

Published: 4 May 2010

\footnotetext{
References

Pospisil P, et al: J Recept Signal Transduct 2003, 23:361-371.

Wolber G, Langer T: J Chem Inf Model 2005, 45:160-169.

Halgren TA: J Comput Chem 1996, 17:490-519.

Halgren TA: J Comput Chem 1996, 17:520-552.

Halgren TA: J Comput Chem 1996, 17:553-586.

Halgren TA, Nachbar RB: J Comput Chem 1996, 17:587-615.

Halgren TA: J Comput Chem 1996, 17:616-651.

Halgren TA: J Comput Chem 1999, 20:720-729.

9. Halgren TA: J Comput Chem 1999, 20:730-748.
}

Inte:Ligand GmbH, Mariahilferstrasse 74B/11, A-1070 Vienna, Austria
doi:10.1186/1758-2946-2-S1-P32

Cite this article as: Seidel and Wolber: Ligand-side tautomer enumeration and scoring for structure-based drug-design. Journal of Cheminformatics 2010 2(Suppl 1):P32.

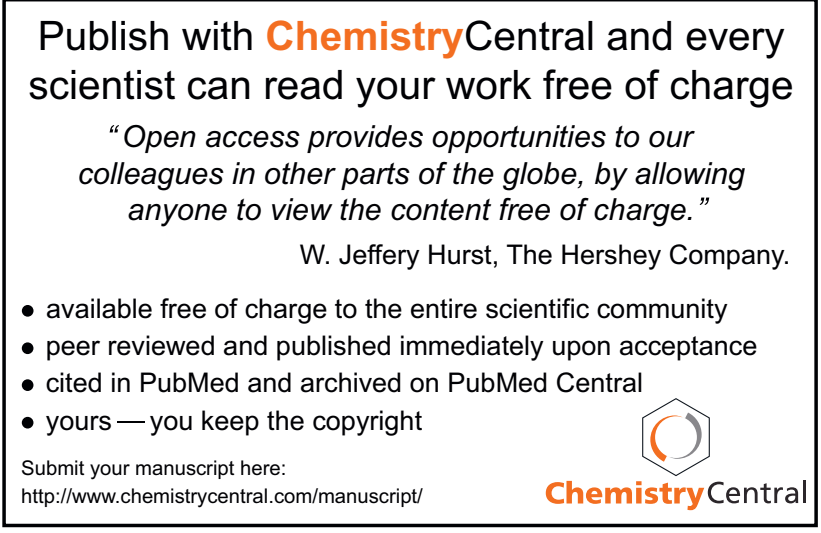

